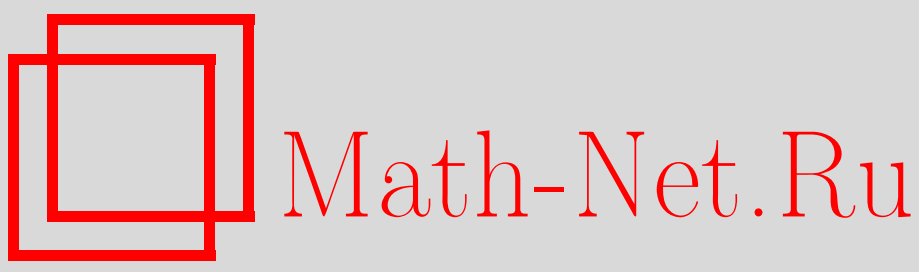

А. М. Седлецкий, Аппроксимативные свойства систем экспонент на прямой и полупрямой, Матем. сб., 1998, том 189, номер 3, 125-140

DOI: https://doi.org/10.4213/sm313

Использование Общероссийского математического портала Math-Net.Ru подразумевает, что вы прочитали и согласны с пользовательским соглашением

http://www . mathnet.ru/rus/agreement

Параметры загрузки:

IP: 54.166 .219 .16

26 апреля 2023 г., 13:18:42 
УДК 517.5

\section{А.М. Седлецкий}

\section{Аппроксимативные свойства систем экспонент на прямой и полупрямой}

\footnotetext{
Для произвольных $a>0, \alpha>1$ строится класс целых функций, зависящих от комплексного параметра $\mu$. Описаны все значения $\mu$, при которых последовательность $\lambda_{n}$ нулей каждой функции этого класса порождает полную и минимальную систему экспонент

$$
\exp \left(-i \lambda_{n} t-a|t|^{\alpha}\right)
$$

в $L^{p}(\mathbb{R})\left(L^{p}\left(\mathbb{R}_{+}\right)\right), p \geqslant 2$. Примеры таких систем были известны раншше только при $\alpha=2$.

Библиограффия: 14 названий.
}

1. Речь пойдет об аппроксимативных свойствах систем экспонент с быстро убывающим весом, а именно, систем вида

$$
e^{-i \lambda_{n} t} \exp \left(-a|t|^{\alpha}\right), \quad \lambda_{n} \in \Lambda ; \quad a>0, \quad \alpha>1
$$

в пространствах $L^{p}$ на всей прямой и на полупрямой.

Первоначально [1]-[5] полнота систем $(1)$ в $L^{2}(\mathbb{R})$ исследовалась для вещественных показателей $\lambda_{n}$. В первую очередь это связано с теоремой Н. Винера [6], по которой линейная оболочка сдвигов

$$
f(t+\lambda)
$$

$(\lambda \in \mathbb{R})$ функции $f \in L^{2}(\mathbb{R})$ плотна в $L^{2}(\mathbb{R})$ тогда и только тогда, когда ее преобразование Фурье $\hat{f} \neq 0$ почти всюду. Эквивалентная формулировка (с $g=\hat{f})$ состоит в том, что линейная оболочка взвешенных экспонент

$$
e^{-i \lambda t} g(t)
$$

$(\lambda \in \mathbb{R})$ плотна в $L^{2}(\mathbb{R})$ тогда и только тогда, когда $g \neq 0$ почти всюду.

Отталкиваясь от этой теоремы, мы приходим к задаче описания последовательностей $\Lambda=\left(\lambda_{n}\right), \lambda_{n} \rightarrow \infty$ (конечно, зависящих от $g, g \neq 0$ почти всюду) таких, что системы (3) с $\lambda \in \Lambda$ представляют собой подходящий аппарат аппроксимации в $L^{2}(\mathbb{R}) . \mathrm{K}$ настоящему моменту теория наиболее продвинута в случае веса

$$
g(t)=\exp \left(-a|t|^{\alpha}\right), \quad a>0, \quad \alpha>1,
$$

т.е. для систем (1).

Работа выполнена при финансовой поддержке Российского фонда фундаментальных исследований (грант № 96-15-96049). 
Пусть $\Lambda \subset \mathbb{C}$, пусть $\beta>1,1 / \alpha+1 / \beta=1$. В статье автора [7] доказано следуюшее. Пусть $\beta$ целое; тогда если система (1) полна и минимальна в $L^{2}(\mathbb{R})$, то

$$
\begin{aligned}
& \bar{\Delta}_{\beta}(\Lambda)>0, \\
& \text { 2) } \varlimsup_{R \rightarrow \infty}\left|\sum_{\left|\lambda_{n}\right|<R} \frac{1}{\lambda_{n}^{\beta}}\right|<\infty .
\end{aligned}
$$

Здесь $\bar{\Delta}_{\beta}(\Lambda)$ обозначает верхнюю плотность последовательности $\Lambda$ при порядке $\beta$, т.е.

$$
\bar{\Delta}_{\beta}(\Lambda)=\varlimsup_{n \rightarrow \infty} \frac{n}{\left|\lambda_{n}\right|^{\beta}} .
$$

Предположим теперь, что $\beta$ целое и $\Lambda \subset \mathbb{R}_{+}$или что $\beta$ четное и $\Lambda \subset \mathbb{R}$. Тогда условие (5) преврашается в условие

$$
\sum \frac{1}{\left|\lambda_{n}\right|^{\beta}}<\infty
$$

влекушее свойство $n /\left|\lambda_{n}\right|^{\beta} \rightarrow 0$ и, в частности, $\bar{\Delta}_{\beta}(\Lambda)=0$. То есть в этом случае условия 1), 2) необходимого признака полноты и минимальности системы (1) в $L^{2}(\mathbb{R})$ несовместимы.

Вывод: если мы хотим иметь дело не только с полньми, но и с минимальньми системами $(1)$ в $L^{2}(\mathbb{R})$, мы должны - с точки зрения расположения последовательности $\Lambda$ - выйти в комплексную плоскость (по крайней мере, в случае четных $\beta$ ).

Отметим, что обратное преобразование Фурье функции (4) есть целая функция, и в эквивалентной постановке речь идет о полных и минимальных в $L^{2}(\mathbb{R})$ системах (2) комплексных с двигов $(\lambda \in \Lambda)$ этой целой функции.

Первыми полную и одновременно минимальную систему $(1)$ в $L^{2}(\mathbb{R})$ с $\alpha=2$ предъявили Р. Залик и Абуабара Т. Саад [8], доказавшие следуюшее. Пусть

$$
\Lambda=\bigcup_{k=0}^{3}\left(2(\pi n)^{1 / 2} \exp \left(i\left(\frac{\pi}{4}+k \frac{\pi}{2}\right)\right)\right)_{n=1}^{\infty} \cup\{ \pm \lambda\}
$$

где $\lambda \neq 0$ не принадлежит предыдушему множеству в (6); тогда система

$$
e^{-i \lambda_{n} t} \exp \left(-t^{2} / 2\right), \quad \lambda_{n} \in \Lambda,
$$

полна и минимальна в $L^{2}(\mathbb{R})$.

Для доказательства утверждения о полноте в [8] был предложен оригинальньй прием; однако в его реализации условие $\alpha=2$ играет существенную роль.

В [9], где рассматривались более общие последовательности

$$
\Lambda=\bigcup_{k=0}^{3}\left(2(\pi(n+h))^{1 / 2} \exp \left(i\left(\frac{\pi}{4}+k \frac{\pi}{2}\right)\right)\right)_{n=1}^{\infty} \cup\{ \pm \lambda\}, \quad h>-1,
$$

установлено, что система $(7),(8)$ полна и минимальна в $L^{2}(\mathbb{R})$ тогда и только тогда, когда $-1 / 8<h \leqslant 1 / 8$. Тем самым предшествуюший результат расширен, но его доказательство использует упомянутый прием из [8], а потому снова проходит только для $\alpha=2$. 
Вопрос о существовании полных и минимальных (одновременно) систем (1) в $L^{2}(\mathbb{R})$ при $\alpha \neq 2$ оставался открытым. Если же говорить о пространстве $L^{2}\left(\mathbb{R}_{+}\right)$, то этот вопрос в литературе вообще не рассматривался, в том числе, и для $\alpha=2$.

В настоящей статье восполняется этот пробел. Для любых $\alpha>1, a>0$ мы конструируем класс целых функций, зависящих от комплексного параметра $\mu$, и описываем все значения $\mu$, при которых последовательность $\Lambda$ нулей каждой функции этого класса порождает полную и минимальную систему $(1)$ в $L^{p}(\mathbb{R})\left(\right.$ в $\left.L^{p}\left(\mathbb{R}_{+}\right)\right)$, $p \geqslant 2$.

2. Всюду в дальнейшем $a>0, \alpha>1, \beta>1,1 / \alpha+1 / \beta=1, p>1, q>1$, $1 / p+1 / q=1, K(\beta, a)=(\alpha a)^{-\beta / \alpha} \cdot \beta^{-1}, z=x+i y=r e^{i \theta}$.

Лемма 1. Пусть $\left|\theta_{k}\right|<\pi, \theta_{k} \neq 0$. Положим

$$
\begin{aligned}
& \theta_{k}^{0}=\frac{\theta_{k}}{\alpha}+\frac{\pi}{2 \beta}, \quad \text { eсли } \quad \theta_{k} \in\left(-\pi,-\frac{\pi}{2}\right] \cup\left(0, \frac{\pi}{2}\right], \\
& \theta_{k}^{0}=\frac{\theta_{k}}{\alpha}-\frac{\pi}{2 \beta}, \quad \text { если } \quad \theta_{k} \in\left(-\frac{\pi}{2}, 0\right) \cup\left(\frac{\pi}{2}, \pi\right) .
\end{aligned}
$$

Введем также следующие обозначения:

$$
\begin{gathered}
a_{k}=\left|\sin \theta_{k}\right|^{\beta-1}, \quad I_{k}=\left(\theta_{k}^{0}-\frac{\pi}{2 \beta}, \theta_{k}^{0}+\frac{\pi}{2 \beta}\right), \\
h_{k}(\theta)=a_{k} \cos \beta\left(\theta-\theta_{k}^{0}\right), \quad \theta \in I_{k} ; \quad h_{k}(\theta)=0, \quad \theta \in[-\pi, \pi] \backslash I_{k}, \\
b_{k}(\theta)=|\sin \theta|^{\beta}-h_{k}(\theta), \quad \theta \in I_{k} .
\end{gathered}
$$

Тогда

1) $h_{k}(\theta) \leqslant|\sin \theta|^{\beta},|\theta| \leqslant \pi$,

2) при $\theta \neq 0, \pm \pi$ знак равенства в 1) достигается в единственной точке $\theta=\theta_{k}$

3) $b_{k}^{\prime}\left(\theta_{k}\right)=0, b_{k}^{\prime \prime}\left(\theta_{k}\right) \neq 0$.

ДокАЗАТЕЛЬСтво. В силу симметрии достаточно рассмотреть случай $0<$ $\theta_{k} \leqslant \pi / 2$. Сначала проверим, что $\theta_{k} \in I_{k}$. По определению $\theta_{k}^{0}$ имеем

$$
\theta_{k}^{0}-\theta_{k}=\frac{\pi}{2 \beta}-\frac{\theta_{k}}{\beta} .
$$

Поэтому $0 \leqslant \theta_{k}^{0}-\theta_{k}<\frac{\pi}{2 \beta}$. И так как $I_{k}$ есть интервал радиуса $\frac{\pi}{2 \beta}$ с центром в точке $\theta_{k}^{0}$, то $\theta_{k} \in I_{k}$.

Теперь убедимся, что $I_{k} \subset(0, \pi)$. Условие $I_{k} \subset(0, \pi)$ равносильно системе неравенств $0<\theta_{k} / \alpha, \theta_{k} / \alpha+\pi / \beta<\pi$, которая записывается в виде двойного неравенства $0<\theta_{k}<\pi$. Это двойное неравенство верно, так как мы рассматриваем случай $0<\theta_{k} \leqslant \pi / 2$. Итак, $I_{k} \subset(0, \pi)$.

Рассмотрим функцию

$$
A(\theta)=\frac{h_{k}(\theta)}{\sin ^{\beta} \theta}, \quad \theta \in I_{k} .
$$

Имеем

$$
\begin{aligned}
& \operatorname{sign} A^{\prime}(\theta)=-\beta a_{k} \sin ^{\beta-1} \theta\left(\sin \beta\left(\theta-\theta_{k}^{0}\right) \sin \theta+\cos \beta\left(\theta-\theta_{k}^{0}\right) \cos \theta\right), \\
& \operatorname{sign} A^{\prime}(\theta)=-\cos \left(\theta(\beta-1)-\beta \theta_{k}^{0}\right)=-\cos (\beta-1)\left(\theta-\alpha \theta_{k}^{0}\right) .
\end{aligned}
$$


Значение $\theta=\theta_{k}$ отвечает значению $-\pi / 2$ под знаком косинуса в (10). Таким образом, $A^{\prime}\left(\theta_{k}\right)=0$, и при переходе через точку $\theta_{k}$ производная $A^{\prime}(\theta)$ меняет знак с плюса на минус. Из (9) следует, что расстояние от точки $\theta_{k}$ до концов интервала $I_{k}$ меньше, чем $\pi / \beta$, и подавно, меньше, чем $\pi /(\beta-1)$. Поэтому правая часть в $(10)$ больше на $I_{k}$ не меняет знака. Следовательно, функция $A(\theta)$ достигает в точке $\theta_{k}$ своего наибольшего значения на $I_{k}$. Но из определений следует, что $A\left(\theta_{k}\right)=1$. Значит, утверждение 1$)$ верно для $\theta \in I_{k}$; для остальных $\theta$ оно очевидно. Из сказанного следует также и утверждение 2 ).

По доказанному точка $\theta_{k}$ есть точка экстремума дифференцируемой функции $b_{k}(\theta)$, и потому $b_{k}^{\prime}\left(\theta_{k}\right)=0$. Далее,

$$
\begin{aligned}
b_{k}^{\prime \prime}(\theta) & =\left(\beta \sin ^{\beta-1} \theta \cos \theta+a_{k} \beta \sin \beta\left(\theta-\theta_{k}^{0}\right)\right)^{\prime} \\
& =\beta\left((\beta-1) \sin ^{\beta-2} \theta \cos ^{2} \theta-\sin ^{\beta} \theta+a_{k} \beta \cos \beta\left(\theta-\theta_{k}^{0}\right)\right) .
\end{aligned}
$$

Но $a_{k} \cos \beta\left(\theta-\theta_{k}^{0}\right)=\sin ^{\beta} \theta$ при $\theta=\theta_{k}$, и значит,

$$
b_{k}^{\prime \prime}(\theta)=\beta(\beta-1) \sin ^{\beta-2} \theta\left(\cos ^{2} \theta+\sin ^{2} \theta\right)=\beta(\beta-1) \sin ^{\beta-2} \theta \neq 0, \quad \theta=\theta_{k} .
$$

Утверждение 3) верно. Лемма 1 доказана.

ЗАмЕчАниЕ. Для дальнейших рассуждений полезно иметь в виду следуюшую геометрическую трактовку леммы 1 . График функции $h_{k}(\theta)$ представляет собой арку косинусоиды, расположенную над интервалом $I_{k}$ длины $\pi / \beta$ с центром в точке $\theta_{k}^{0}$. Эта арка вписана в график функции $|\sin \theta|^{\beta}$ так, что точка $\theta_{k}$ является точкой касания этих двух графиков, а для остальных значений $\theta \in I_{k}$ график функции $h_{k}(\theta)$ лежит ниже графика функции $|\sin \theta|^{\beta}$.

Заметим еще, что обозначения леммы 1 , равно как и обозначения леммы 2 , используются в дальнейшем без дополнительных напоминаний.

Лемма 2. Пусть $-\pi<\theta_{1}<\theta_{2}<\cdots<\theta_{m}<\pi, \theta_{k} \neq 0, k=\overline{1, m}$. Обозначим

$$
\begin{aligned}
h(\theta) & =\max \left(h_{k}(\theta): k=\overline{1, m}\right), & & |\theta| \leqslant \pi, \\
b(\theta) & =|\sin \theta|^{\beta}-h(\theta), & & |\theta| \leqslant \pi .
\end{aligned}
$$

Тогда

1) $h(\theta) \leqslant|\sin \theta|^{\beta},|\theta| \leqslant \pi$,

2) знак равенства при $\theta \neq 0, \pm \pi$ в 1) достигается в точках $\theta_{k}, k=\overline{1, m}$, и только в них,

3) $b(\theta) \sim c_{k}\left(\theta-\theta_{k}\right)^{2}, \theta \rightarrow \theta_{k}, c_{k} \neq 0, k=\overline{1, m}$.

ДокАЗАТЕЛЬСТво. Вне объединения интервалов $I_{k}$ имеем $h(\theta)=0$, и для таких $\theta$ утверждение 1$)$ очевидно. График функции $h_{k}(\theta)$ есть арка косинусоиды, расположенная над интервалом $I_{k}$, а график функции $h(\theta)$ получается склейкой частей граффиков функций $h_{k}(\theta)$. Так как $\theta_{k}<\theta_{k+1}$, то при этом у каждой точки $\theta_{k}$ существует окрестность, в которой $h(\theta)=h_{k}(\theta)$, причем замькание объединения этих окрестностей совпадает с замьканием объединения интервалов $I_{k}$. Поэтому оставшаяся часть леммы 2 следует из леммы 1 . Лемма 2 верна.

В дальнейшем важную роль будут играть следуюшие понятия: тригонометрически $\rho$-выпуклая функция, индикатор целой функции, целая функция вполне регулярного роста. По поводу этих понятий см., например, [10]. 
Лемма 3. Пусть $H(\theta)$ - тригонометрически $\beta$-выпуклая функция, $\theta_{1} \leqslant$ $\theta \leqslant \theta_{3}$, причем $H\left(\theta_{i}\right) \leqslant 0, i=1,3$. Тогда

1) если $\theta_{3}-\theta_{1}<\pi / \beta$, то $H(\theta) \leqslant 0$ на $\left[\theta_{1}, \theta_{3}\right]$,

2) если $\theta_{3}-\theta_{1}=\pi / \beta$ и $H\left(\theta_{i}\right)<0$ хотя бъ при одном значении $i=1,3$, то $H(\theta) \leqslant 0$ на $\left[\theta_{1}, \theta_{3}\right]$.

ДокаЗАТЕЛЬСТво. Можно считать, что $\theta_{1}=0$. Фиксируем $\varepsilon>0$ и рассмотрим $\beta$-тригонометрическую функцию $t(\theta)=\varepsilon \sin \beta \theta$.

1) Так как $0<\beta \theta_{3}<\pi$, то $H(0) \leqslant t(0), H\left(\theta_{3}\right)<t\left(\theta_{3}\right)$. По определению тригонометрической $\beta$-выпуклости отсюда следует, что

$$
H(\theta) \leqslant t(\theta)=\varepsilon \sin \beta \theta, \quad 0 \leqslant \theta \leqslant \theta_{3} .
$$

Так как $\varepsilon$ здесь можно взять сколь угодно малым, то $H(\theta) \leqslant 0, \theta_{1} \leqslant \theta \leqslant \theta_{3}$.

2) Так как $\theta_{1}=0$, то $\theta_{3}=\pi / \beta$. Пусть для определенности $H(0)<0$. В силу непрерывности тригонометрически $\beta$-выпуклой функции имеем $H\left(\theta_{2}\right)<t\left(\theta_{2}\right)$ при достаточно малом $\theta_{2} \in(0, \pi / \beta)$. Значит,

$$
H(0)<t(0), \quad H\left(\theta_{2}\right)<t\left(\theta_{2}\right), \quad H(\pi / \beta) \leqslant t(\pi / \beta) .
$$

По определению тригонометрической $\beta$-выпуклости из первой пары этих неравенств следует, что $H(\theta) \leqslant t(\theta)$ на $\left[0, \theta_{2}\right]$, а из второй пары следует, что $H(\theta) \leqslant t(\theta)$ на $\left[\theta_{2}, \pi / \beta\right]$. В итоге мы получаем неравенство (11) с $\theta_{3}=\pi / \beta$, откуда $H(\theta) \leqslant 0$, $0 \leqslant \theta \leqslant \theta_{3}=\pi / \beta$. Лемма 3 доказана.

Лемма 4. Пусть $H(\theta)$ - тригонометрически $\beta$-вьпуклая функиия. Пусть $\theta_{1}<\theta_{3}, \theta_{3}-\theta_{1}=\pi / \beta, H\left(\theta_{1}\right)=H\left(\theta_{3}\right)=0$ и $H(\theta)=o\left(\theta-\theta_{i}\right)$ хотя би при одном значении $i=1,3$. Тогда $H(\theta) \leqslant 0, \theta_{1} \leqslant \theta \leqslant \theta_{3}$.

ДоказАТЕЛЬСтво. Снова считаем, что $\theta_{1}=0, \theta_{3}=\pi / \beta$. Пусть для определенности $H(\theta)=o(\theta), \theta \rightarrow+0$. Пусть функция $t(\theta)$ та же, что в доказательстве леммы 3. Так как $\sin \beta \theta \sim \beta \theta$, а $H(\theta)=o(\theta), \theta \rightarrow+0$, то при достаточно малом $\theta_{2} \in(0, \pi / \beta)$ будем иметь $H\left(\theta_{2}\right)<t\left(\theta_{2}\right)$. То есть

$$
H(0)=t(0), \quad H\left(\theta_{2}\right)<t\left(\theta_{2}\right), \quad H(\pi / \beta)=t(\pi / \beta) .
$$

Теперь целиком повторяются рассуждения, следующие за (12). Лемма 4 доказана.

Лемма 5. Пусть $b(\theta)$ - функиия из леммы 2, a $A, \beta>0, S_{k}=(z: r>1$, $\left.\left|\theta-\theta_{k}\right|<\delta\right)$. Тогда если $\delta>0$ достаточно мало, то условие

$$
J_{k}=\iint_{S_{k}} \exp \left(-A b(\theta) r^{\beta}\right) r^{s} d r d \theta<\infty \quad(s \in \mathbb{R})
$$

равносильно условию $s+1<\beta / 2$. 
ДокаЗАТЕЛЬСТво. По лемме $2 b(\theta)>0$ при $\theta \in U_{k}=\left(\theta:\left|\theta-\theta_{k}\right|<\delta\right), \theta \neq \theta_{k}$; $b\left(\theta_{k}\right)=0$. Положим $t=b(\theta) r^{\beta}$. Тогда

$$
r=(t / b(\theta))^{1 / \beta}, \quad d r=(1 / \beta) t^{1 / \beta-1}(b(\theta))^{-1 / \beta} d t,
$$

и с точностью до ненулевого постоянного сомножителя

$$
J_{k}=\int_{U_{k}} \frac{d \theta}{(b(\theta))^{(s+1) / \beta}} \int_{b(\theta)}^{\infty} e^{-A t} t^{(s+1) / \beta-1} d t .
$$

Пусть сначала $s+1>0$. Тогда обозначив $b=\max \left(b(\theta): \theta \in \bar{I}_{k}\right)$, имеем

$$
0<c_{1}=\int_{b}^{\infty}<\int_{b(\theta)}^{\infty}<\int_{0}^{\infty}=c_{2}<\infty
$$

и вспоминая лемму 2 , видим, что интеграл $J_{k}$ конечен или бесконечен одновременно с интегралом

$$
\int_{0}^{\infty} \theta^{-2(s+1) / \beta} d \theta
$$

Конечность последнего интеграла равносильна условию $2(s+1) / \beta<1$. Таким образом, если $s+1>0$, то условие $J_{k}<\infty$ равносильно условию $s+1<\beta / 2$. Поэтому остается показать, что если $s+1 \leqslant 0$, то $J_{k}<\infty$.

Но это уже следует из доказанной части. Действительно, так как $r>1$ для $z \in S_{k}$, то из определения (13) интеграла $J_{k}$ видно, что если он конечен при некотором значении $s=s_{0}$, то он будет конечным и при всех меншшх значениях $s$, т.е. при $s \leqslant s_{0}$. Лемма 5 доказана.

Лемма 6. Пусть $\sigma_{1}=(z: r>1,0<\theta<\delta), \sigma_{2}=(z: r>1, \pi-\delta<\theta<\pi)$, $\delta>0, s \in \mathbb{R}, q>1, A>0, \beta>0$. Тогда

$$
\iint_{\sigma_{i}}(1+y)^{s} \exp \left(-A y^{\beta}\right) r^{-q} d x d y<\infty, \quad i=1,2 .
$$

ДокАЗАТЕльСтво. Интеграл в (14) не зависит от $i$; разберем случай $i=1$. Пусть $\pi$ - пересечение $\sigma_{1}$ с полосой $0<y<1$. Сначала докажем, что конечна часть интеграла (14), взятая по $\pi$. Имеем

$$
(1+y)^{s} \exp \left(-A y^{\beta}\right) \leqslant C<\infty, \quad z \in \pi,
$$

и потому, если $\varepsilon=1 / \sin \delta, a(r)=\arcsin (1 / r)$, то

$$
\iint_{\pi} \leqslant C \iint_{\pi} r^{-q} d x d y \leqslant c_{1}+c_{2} \int_{\varepsilon}^{\infty} r^{-q+1} d r \int_{0}^{a(r)} d \theta \leqslant c_{1}+c_{3} \int_{\varepsilon}^{\infty} r^{-q} d r<\infty
$$

так как $q>1$.

Остается рассмотреть ту часть интеграла в (14), которая берется по множеству $\sigma=\left(z \in \sigma_{1}: y \geqslant 1\right)$.

Если $z \in \sigma$, то $1+y \asymp y$, и значит,

$$
\iint_{\sigma}<c \int_{\varepsilon}^{\infty} r^{-q+s+1} d r \int_{a(r)}^{\delta} \theta^{s} \exp \left(-B(\theta r)^{\beta}\right) d \theta, \quad B>0
$$


Положим $(\theta r)^{\beta}=t$; тогда $\theta=t^{1 / \beta} / r, d \theta=c t^{1 / \beta-1} d t / r$, и внутренний интеграл в (15) не превосходит

$$
\frac{c}{r^{s+1}} \int_{\Delta}^{\infty} e^{-B t} t^{(s+1) / \beta-1} d t \leqslant \frac{c_{1}}{r^{s+1}}, \quad \Delta=\left(r \arcsin \frac{1}{r}\right)^{\beta} \geqslant \Delta_{1}>0 .
$$

Значит,

$$
\iint_{\sigma} \leqslant c \int_{\varepsilon}^{\infty} r^{-q} d r<\infty
$$

и лемма 6 доказана.

В наших построениях важную роль играет функция типа Миттаг-Леффлера [11]

$$
E_{\beta}(z ; \mu)=\sum_{n=0}^{\infty} \frac{z^{n}}{\Gamma(\mu+n / \beta)}, \quad \beta>0, \quad \mu \in \mathbb{C} ;
$$

$E_{\beta}(z ; \mu)$ - целая функция.

Teорема A [11]. Если $\gamma \in(\pi /(2 \beta), \pi), \beta>1 / 2$, то при любом $s \in \mathbb{N}$

$$
\begin{array}{ll}
E_{\beta}(z ; \mu)=\beta z^{\beta(1-\mu)} \exp \left(z^{\beta}\right)-\sum_{j=1}^{s} \frac{z^{-j}}{\Gamma(\mu-j / \beta)}+O\left(\frac{1}{r^{s+1}}\right), & |\arg z| \leqslant \gamma, \\
E_{\beta}(z ; \mu)=-\sum_{j=1}^{s} \frac{z^{-j}}{\Gamma(\mu-j / \beta)}+O\left(\frac{1}{r^{s+1}}\right), & \gamma \leqslant|\arg z| \leqslant \pi .
\end{array}
$$

Нам понадобятся также свойства преобразований Фурье быстро убываюших функций. Пусть

$$
G(z)=\int_{\mathbb{R}} e^{-i z t} \exp \left(-a|t|^{\alpha}\right) g(t) d t
$$

Теорема Б [12]. Если $g(t) \in L^{q}(\mathbb{R}), q \geqslant 1$, то $G(z)$ - целая функция и при всех $\theta \neq \pi n \quad(n \in \mathbb{Z})$

$$
\int_{1}^{\infty}\left|G\left(r e^{i \theta}\right)\right|^{q} r^{(q-2)(1-\beta / 2)} \exp \left(-q K(\beta, a) r^{\beta}|\sin \theta|^{\beta}\right) d r<\infty .
$$

Tеорема В [12]. Пусть $1<q \leqslant 2.1)$ Пусть $G(z)$ - челая функция такая, чmo

$$
\iint_{\mathbb{R}^{2}}|G(z)|^{q}(1+|y|)^{(q-3)(1-\beta / 2)} \exp \left(-q K(\beta, a)|y|^{\beta}\right) d x d y<\infty .
$$

Тогда $G(z)$ представима в виде $(16)$ с $g(t) \in L^{q}(\mathbb{R})$.

2) Пусть $G(z)$ - целая функиия такая, что $G(z) \in H_{-}^{2}$ и конечен интеграл в (18), взятый по полуплоскости $у>0$. Тогда $G(z)$ представима в виде (16) с $g(t) \in L^{q}(\mathbb{R})$, причем $g(t)=0$ при $t<0$.

Здесь через $H_{-}^{2}$ обозначен класс Харди в нижней полуплоскости, т.е. класс функций, аналитических при $y<0$ и таких, что

$$
\sup _{y<0} \int_{\mathbb{R}}|F(x+i y)|^{2} d x<\infty .
$$


3. Полнота и неминимальность систем экспонент на прямой и на полупрямой.

Теорема 1. Пусть $-\pi<\theta_{1}<\cdots<\theta_{l}<0<\theta_{l+1}<\cdots<\theta_{m}<\pi$, причем

$$
\begin{gathered}
\theta_{1}+\pi \leqslant \frac{\pi}{\beta}, \quad \theta_{l} \geqslant-\frac{\pi}{\beta}, \quad \theta_{l+1} \leqslant \frac{\pi}{\beta}, \quad \pi-\theta_{m} \leqslant \frac{\pi}{\beta}, \\
\theta_{k+1}-\theta_{k} \leqslant \frac{\pi}{\beta}, \quad k=1, \ldots, l-1, l+1, \ldots, m-1 .
\end{gathered}
$$

Положим

$$
e(z)=\sum_{k=1}^{m} \pm a_{k} E_{\beta}\left(z e^{-i \theta_{k}^{0}} ; \mu\right), \quad \mu \in \mathbb{C},
$$

әде числа $\theta_{k}^{0}$ и $a_{k}$ определены в лемме 1 , а знаки в (19) выбраны так, что

$$
\begin{array}{lll}
\sum_{k=1}^{m} \pm a_{k} e^{i \theta_{k}^{0}} \neq 0, & \text { если } & \mu-\frac{1}{\beta} \notin\{0,-1,-2, \ldots\}, \\
\sum_{k=1}^{m} \pm a_{k} e^{2 i \theta_{k}^{0}} \neq 0, & \text { если } \quad \mu-\frac{1}{\beta} \in\{0,-1,-2, \ldots\} .
\end{array}
$$

Обозначим

$$
F(z)=e\left((K(\beta, a))^{1 / \beta} z\right),
$$

и пусть $\Lambda$ - последовательность всех нулей функций $F(z)$. Пусть $1<p<\infty$. Тогда

1) ecлu

$$
\operatorname{Re} \mu \leqslant \frac{1}{\beta}+\frac{1}{q \alpha}+\frac{1}{2}
$$

то система (1) полна в $L^{p}(\mathbb{R})$,

2) если

$$
\operatorname{Re} \mu \leqslant \frac{1}{q \alpha}+\frac{1}{2}
$$

то система (1) неминимальна в $L^{p}(\mathbb{R})$.

ЗАмечАниЕ. Нулю $\lambda$ функции $F(z)$ кратности $s$ в системе (1) отвечают $s$ функций

$$
t^{j} e^{-i \lambda t} \exp \left(-a|t|^{\alpha}\right), \quad j=\overline{0, s-1} .
$$

ДокАЗАТЕЛЬСТво тЕОРЕмЫ 1. 1) Предположим, что система (1) неполна в $L^{p}(\mathbb{R})$, и докажем, что

$$
\operatorname{Re} \mu>\frac{1}{\beta}+\frac{1}{q \alpha}+\frac{1}{2} .
$$

Так как система $(1)$ неполна в $L^{p}(\mathbb{R})$, то найдется нетривиальная функция $g(t) \in L^{q}(\mathbb{R})$ такая, что целая функция (16) обрашается в 0 в точках $\Lambda$. Но $\Lambda-$ последовательность всех нулей функции $F(z)$. Поэтому

$$
G(z)=F(z) E(z)
$$

где $E(z)$ - некоторая целая функция. Покажем, что $E(z) \in[\beta, 0]$, т.е. что $E(z)$ имеет минимальный тип при порядке $\beta$. 
Из (16) следует, что

$$
|G(z)| \leqslant M<\infty, \quad|y| \leqslant 1 .
$$

Далее (см. [12]) справедлива оценка

$$
|G(z)| \leqslant C|y|^{\beta / 2-1} \exp \left(K(\beta, a)|y|^{\beta}\right), \quad|y| \geqslant 1 .
$$

Из $(27)$ и (28) следует, что порядок функции $G(z)$ не вьше $\beta$ и что ее индикатор $h_{G}(\theta)$ при порядке $\beta$ подчиняется оценке

$$
h_{G}(\theta) \leqslant K(\beta, a)|\sin \theta|^{\beta}, \quad|\theta| \leqslant \pi .
$$

Перейдем к функции $F(z)$. По теореме А порядок функции $E_{\beta}(z ; \mu)$ равен $\beta$, а ее индикатор равен:

$$
\begin{array}{ll}
\cos \beta \theta & \text { при }|\theta| \leqslant \frac{\pi}{2 \beta}, \\
0 & \text { при }|\theta| \geqslant \frac{\pi}{2 \beta} .
\end{array}
$$

Из теоремы А также следует, что $E_{\beta}(z ; \mu)$ есть целая функция вполне регулярного роста.

Отсюда делаем вывод, что функция $a_{k} E_{\beta}\left(z e^{-i \theta_{k}^{0}} ; \mu\right)$ имеет вполне регулярный рост и что ее индикатор равен $h_{k}(\theta),|\theta| \leqslant \pi$.

Теперь напомним следуюшие два свойства индикатора $\left(h_{F}(\theta)\right.$ обозначает индикатор функции $F(z)$ ).

Если $h_{F_{1}}(\theta)>h_{F_{2}}(\theta)$, то $h_{F_{1}+F_{2}}(\theta)=h_{F_{1}}(\theta)$.

Если при этом луч $\arg z=\theta$ является лучом вполне регулярного роста для функции $F_{1}(z)$, то он является таким и для функции $F_{1}(z)+F_{2}(z)$.

Учитьвая эти свойства, вид (19) функции $e(z)$ и только что сказанное о росте функции $a_{k} E_{\beta}\left(z e^{-i \theta_{k}^{0}} ; \mu\right)$, получаем следуюшее. Во-первых, индикатор $h_{e}(\theta)$ функции $е(z)$ равен

$$
h_{e}(\theta)=h(\theta), \quad \theta \in \bigcup I_{k}, \quad \theta \notin T,
$$

где $T$ - множество точек недифференцируемости функции $h(\theta)$. Во-вторых, все лучи $\arg z=\theta, \theta \in \bigcup I_{k}, \theta \notin T$, являются лучами вполне регулярного роста функции $е(z)$.

В силу непрерывности индикатора

$$
h_{e}(\theta)=h(\theta), \quad \theta \in \bigcup \bar{I}_{k}
$$

Выясним поведение $e(z)$ на лучах $\arg z=\theta \notin \bigcup \bar{I}_{k}$. В силу теоремы А при любом натуральном $s$

$$
a_{k} E_{\beta}\left(z e^{-i \theta_{k}^{0}} ; \mu\right)=a_{k} \sum_{j=1}^{s} \frac{z^{-j} e^{i j \theta_{k}^{0}}}{\Gamma(\mu-j / \beta)}+O\left(\frac{1}{r^{s+1}}\right), \quad \arg z=\theta \notin \bar{I}_{k} .
$$

Значит, беря $s=2$, при $\theta \notin \bigcup \bar{I}_{k}$ получаем

$$
e(z)=\frac{z^{-1}}{\Gamma(\mu-1 / \beta)} \sum_{k=1}^{m} \pm a_{k} e^{i \theta_{k}^{0}}+\frac{z^{-2}}{\Gamma(\mu-2 / \beta)} \sum_{k=1}^{m} \pm a_{k} e^{2 i \theta_{k}^{0}}+O\left(\frac{1}{r^{3}}\right) .
$$


Если $\mu-1 / \beta \neq 0,-1, \ldots$, то $\frac{1}{\Gamma(\mu-1 / \beta)} \neq 0$ и тогда

$$
e(z)=\frac{c}{z}+O\left(\frac{1}{r^{2}}\right), \quad r \rightarrow \infty, \quad \arg z \notin \bigcup \bar{I}_{k},
$$

где $c \neq 0$ в силу (20). Если же $\mu-1 / \beta \in\{0,-1, \ldots\}$, то $\frac{1}{\Gamma(\mu-1 / \beta)}=0$, но $\frac{1}{\Gamma(\mu-2 / \beta)} \neq 0$, так как $0<1 / \beta<1$, и потому $\mu-1 /(2 \beta) \neq 0,-1, \ldots$ Тогда

$$
e(z)=\frac{c}{z^{2}}+O\left(\frac{1}{r^{3}}\right), \quad r \rightarrow \infty, \quad \arg z \notin \bigcup \bar{I}_{k},
$$

где $c \neq 0$ в силу (21). В любом из этих случаев

$$
h_{e}(\theta)=0, \quad \theta \notin \bigcup \bar{I}_{k},
$$

причем в соответствующих секторах $e(z)$ имеет вполне регулярный рост.

Отсюда вытекают необходимые нам свойства функции $F(z)$ (см. (22)): все лучи $\arg z=\theta \notin T$, где $T$ - множество точек недифференцируемости функции $h(\theta)$, являются лучами вполне регулярного роста функции $F(z)$, и ее индикатор равен

$$
h_{F}(\theta)=K(\beta, a) h(\theta), \quad|\theta| \leqslant \pi .
$$

Теперь мы можем заняться непосредственно функцией $E(z)$. Напомним только еще, что если луч $\arg z=\theta$ является лучом вполне регулярного роста хотя бы для одной из функций $F_{1}(z), F_{2}(z)$, то

$$
h_{F_{1} F_{2}}(\theta)=h_{F_{1}}(\theta)+h_{F_{2}}(\theta) .
$$

С учетом этого и сказанного чуть выше о росте $F(z)$, из $(26),(29)$ заключаем, что

$$
h_{E}(\theta) \leqslant K(\beta, a) b(\theta), \quad b(\theta)=|\sin \theta|^{\beta}-h(\theta), \quad|\theta| \leqslant \pi .
$$

Нам надо убедиться, что $h_{E}(\theta) \leqslant 0$ всюду; тогда мы будем уверены, что $E(z) \in[\beta, 0]$.

Функция $h_{E}(\theta)$ является тригонометрически $\beta$-вьпуклой.

Присоединим к точкам $\theta_{1}, \ldots, \theta_{m}$ точки $0, \pm \pi$. Объединенную систему обозначим $\widehat{\theta}_{k}, k=\overline{0, m+2} ;$ считаем, что она пронумерована так:

$$
-\pi=\widetilde{\theta}_{0}<\tilde{\theta}_{1}<\cdots<\tilde{\theta}_{m+1}<\widetilde{\theta}_{m+2}=\pi
$$

По условию $\widetilde{\theta}_{k+1}-\widetilde{\theta}_{k} \leqslant \pi / \beta, k=\overline{0, m+1}$. Покажем, что

$$
h_{E}(\theta) \leqslant 0, \quad \tilde{\theta}_{k} \leqslant \theta \leqslant \widetilde{\theta}_{k+1}, \quad k=\overline{0, m+1} .
$$

Так как $\left(\widetilde{\theta}_{k}\right)=\left(\theta_{k}\right) \cup\{0, \pm \pi\}$, то из $(34)$ и леммы 2 следует, что $h\left(\widetilde{\theta}_{k}\right) \leqslant 0$, $k=\overline{0, m+2}$. Если $\widetilde{\theta}_{k+1}-\widetilde{\theta}_{k}<\pi / \beta$, то $h_{E}(\theta) \leqslant 0$ на $\left[\widetilde{\theta}_{k}, \widetilde{\theta}_{k+1}\right]$ по первому утверждению леммы 3 . Поэтому остается рассмотреть случай $\widetilde{\theta}_{k+1}-\widetilde{\theta}_{k}=\pi / \beta$. Если $h_{E}\left(\widetilde{\theta}_{k}\right)<0$ или $h_{E}\left(\widetilde{\theta}_{k+1}\right)<0$, то $h_{E}(\theta) \leqslant 0, \widetilde{\theta}_{k} \leqslant \theta \leqslant \widetilde{\theta}_{k+1}$ по второму 
утверждению леммы 3 . Если же $h_{E}\left(\widetilde{\theta}_{k}\right)=h_{E}\left(\widetilde{\theta}_{k+1}\right)=0$, то в силу $(34)$ из леммы 2 следует, что

$$
h_{E}(\theta)=O\left(\left(\theta-\widetilde{\theta}_{j}\right)^{2}\right), \quad \theta \rightarrow \widetilde{\theta}_{j}, \quad j=k, k+1 .
$$

По лемме $4 h_{E}(\theta) \leqslant 0$ на $\left[\widetilde{\theta}_{k}, \widetilde{\theta}_{k+1}\right]$ и в этом случае. Мы доказали (35). Значит, $h_{E}(\theta) \leqslant 0,|\theta| \leqslant \pi$, и следовательно, $E(z) \in[\beta, 0]$.

Следуюший шаг состоит в том, чтобы доказать, что $E(z)$ есть многочлен. Для этого выясним поведение $E(z)$ на лучах $\arg z=\widetilde{\theta}_{k}, k=\overline{1, m+2}$.

Пусть $U_{k}$ - достаточно малая окрестность точки $\theta_{k}$. В силу теоремы А равномерно по $\theta \in U_{k}$

$$
|F(z)| \sim C r^{\beta(1-\operatorname{Re} \mu)} \exp \left(K(\beta, a) r^{\beta} h(\theta)\right), \quad C>0, \quad r \rightarrow \infty .
$$

Отсюда и из (28) с учетом того, что $h\left(\theta_{k}\right)=\left|\sin \theta_{k}\right|^{\beta}$, находим

$$
|E(z)| \leqslant C r^{\beta \operatorname{Re} \mu-\beta / 2-1}, \quad r \rightarrow \infty, \quad \arg z=\theta_{k}, \quad k=\overline{1, m} .
$$

Из (27), (31), (32) видно, что

$$
|E(z)|=O\left(r^{2}\right), \quad r \rightarrow \infty, \quad \arg z=0, \pm \pi .
$$

Таким образом, на лучах $\arg z=\widetilde{\theta}_{k}, k=\overline{1, m+2}$ функция $E(z)$ растет не быстрее степени. Напомним, что $E(z) \in[\beta, 0]$ и что $\widetilde{\theta}_{k+1}-\widetilde{\theta}_{k} \leqslant \pi / \beta, k=\overline{0, m+1}$. Применяя теорему Фрагмена-Линделёфа к секторам $\widetilde{\theta}_{k} \leqslant \arg z \leqslant \widetilde{\theta}_{k+1}, k=$ $\overline{0, m+1}$, заключаем, что в каждом из них, а значит, и во всей плоскости функция $E(z)$ растет не быстрее степени. В итоге $E(z)$ есть многочлен.

Какова бы ни была степень этого многочлена,

$$
|E(z)| \geqslant C>0, \quad r>r_{0} .
$$

Используя это, $(26),(36)$, находим, что при всех $k=\overline{1, m}$

$$
\left|G\left(r e^{i \theta}\right)\right| \geqslant c r^{\beta(1-\operatorname{Re} \mu)} \exp \left(K(\beta, a) r^{\beta} h(\theta)\right), \quad c>0, \quad r>r_{0}, \quad \theta=\theta_{k} .
$$

Но по теореме Б для функции $G(z)$ должно выполняться условие (17) при $\theta=\theta_{k}$. И так как $h\left(\theta_{k}\right)=\left|\sin \theta_{k}\right|^{\beta}$, то отсюда

$$
\int^{\infty} r^{(q-2)(1-\beta / 2)+q \beta(1-\operatorname{Re} \mu)} d r<\infty .
$$

Значит, $(q-2)(1-\beta / 2)+q \beta(1-\operatorname{Re} \mu)<-1$, что равносильно условию (25). Утверждение 1) доказано.

2) Пусть система $(1)$ минимальна в $L^{p}(\mathbb{R})$. Пусть $g(t) \in L^{q}(\mathbb{R})$ та функция биортогональной системы, которая аннулирует функции $(1)$ при $n \neq 0$. Тогда функция (16) обрашается в 0 в точках $\Lambda \backslash \lambda_{0}$, и потому

$$
G(z)=\frac{E(z) F(z)}{z-\lambda_{0}},
$$

где $E(z)$ - некоторая целая функция. Как и при доказательстве утверждения 1$)$, убеждаемся сначала, что $E(z) \in[\beta, 0]$, а затем, что $E(z)$ - многочлен. Значит,

$$
|G(z)| \geqslant \frac{c|F(z)|}{r}, \quad c>0, \quad r>r_{0},
$$


и в силу (36)

$$
\left|G\left(r e^{i \theta}\right)\right| \geqslant c r^{\beta(1-\operatorname{Re} \mu)-1} \exp \left(K(\beta, a) r^{\beta} h(\theta)\right), \quad c>0, \quad r>r_{0}, \quad \theta=\theta_{k} .
$$

Так как $h\left(\theta_{k}\right)=\left|\sin \theta_{k}\right|^{\beta}$, то отсюда по теореме Б

$$
\int^{\infty} r^{(q-2)(1-\beta / 2)+q \beta(1-\operatorname{Re} \mu)-q} d r<\infty .
$$

Следовательно, $(q-2)(1-\beta / 2)+q \beta(1-\operatorname{Re} \mu)-q<-1$, и значит, $\operatorname{Re} \mu>1 / 2+$ $1 /(q \alpha)$, что противоречит предположению. Мы доказали утверждение 2) и всю теорему 1.

Теорема 2. Пусть $0<\theta_{1}<\cdots<\theta_{m}<\pi$, причем

$$
\theta_{1} \leqslant \frac{\pi}{\beta}, \quad \theta_{k+1}-\theta_{k} \leqslant \frac{\pi}{\beta}, \quad k=\overline{1, m-1}, \quad \pi-\theta_{m} \leqslant \frac{\pi}{\beta} .
$$

Пусть е(z) - функция (19), где числа $\theta_{k}^{0}$ и $a_{k}$ определень в лемме 1 , а знаки выбраны в соответствии с условиями (20) и (21). Пусть $F(z)$ - функиия (22), и $\Lambda$ - последовательность всех ее нулей. Пусть $1<p<\infty$. Тогда

1) если выполнено условие (23), то система (1) полна в $L^{p}\left(\mathbb{R}_{+}\right)$,

2) если выполнено условие (24), то система $(1)$ неминимальна в $L^{p}\left(\mathbb{R}_{+}\right)$.

ДокАЗАТЕльство. Предположим, что система (1) неполна в $L^{p}\left(\mathbb{R}_{+}\right)$, и покажем, что имеет место условие (25). По предположению найдется нетривиальная функция $g(t) \in L^{q}(\mathbb{R}), g(t)=0$ при $t<0$, такая, что целая функция (16) обращается в 0 в точках $\Lambda$. Отсюда следует $(26)$, где $E(z)$ - целая функция. Надо показать, что $E(z) \in[\beta, 0]$. Для $G(z)$ оценка (28) сохраняется при $y \geqslant 1$, а оценка $(27)$ верна при $y \leqslant 1$, так как $g(t)=0$ при $t<0$. Значит, порядок $G(z)$ не выше $\beta$, а для ее индикатора верны оценки

$$
h_{G}(\theta) \leqslant K(\beta, a) \sin ^{\beta} \theta, \quad 0 \leqslant \theta \leqslant \pi ; \quad h_{G}(\theta) \leqslant 0, \quad-\pi \leqslant \theta \leqslant 0 .
$$

Далее, повторяя рассуждения из доказательства теоремы 1 , заключаем, что индикатор $h_{e}(\theta)$ функции $e(z)$ при порядке $\beta$ равен

$$
h_{e}(\theta)=h(\theta), \quad 0 \leqslant \theta \leqslant \pi ; \quad h(\theta)=0, \quad-\pi \leqslant \theta \leqslant 0,
$$

и что $e(z)$ имеет вполне регулярный рост на всех лучах, за исключением, быть может, лучей $\arg z=\theta \in T$, где $T$ - множество точек недифференцируемости функции $h(\theta), 0<\theta<\pi$. Отсюда следует, что индикатор функции $F(z)$ равен

$$
h_{F}(\theta)=K(\beta, a) h(\theta), \quad 0 \leqslant \theta \leqslant \pi ; \quad h_{F}(\theta)=0, \quad-\pi \leqslant \theta \leqslant 0,
$$

и что лучи $\arg z=\theta \notin T$ являются лучами вполне регулярного роста функции $F(z)$. Тогда для индикатора функции $E(z)$ верны оценки

$$
h_{E}(\theta) \leqslant K(\beta, a) b(\theta), \quad 0 \leqslant \theta \leqslant \pi ; \quad h_{E}(\theta) \leqslant 0, \quad-\pi \leqslant \theta \leqslant 0 .
$$

Надо доказать, что $h_{E}(\theta) \leqslant 0$ при $0 \leqslant \theta \leqslant \pi$. Это делается точно так же, как в доказательстве теоремы 1 . Сначала к набору $\theta_{1}, \ldots, \theta_{m}$ присоединяются точки $0, \pi$. Затем по аналогии устанавливается, что в секторах $0 \leqslant \arg z \leqslant \theta_{1}, \ldots$, $\theta_{m} \leqslant \arg z \leqslant \pi E(z)$ растет не быстрее степени. Это утверждение для нижней полуплоскости следует из оценки (27) при $y \leqslant 0$, из равенства (26) и из (31), (32). На этом шаге получается, что $E(z)$ - многочлен. Заключительный этап - вывод условия (25) из теоремы Б - осуществляется так же, как в теореме 1. Утверждение 1) доказано.

Так же по аналогии с доказательством утверждения 2) теоремы 1 проводится доказательство утверждения 2) теоремы 2. Теорема 2 доказана. 


\section{4. Неполнота и минимальность систем экспонент на прямой и на} полупрямой.

TеОРема 3. Пусть функиия $F(z)$ та же, что в теореме 1 , пусть $\Lambda$ последовательность всех нулей функиии $F(z)$. Пусть $2 \leqslant p<\infty$. Тогда

1) если

$$
\operatorname{Re} \mu>\frac{1}{\beta}+\frac{1}{q \alpha}+\frac{1}{2}
$$

то система (1) неполна в $L^{p}(\mathbb{R})$,

2) если

$$
\operatorname{Re} \mu>\frac{1}{q \alpha}+\frac{1}{2}
$$

то система (1) минимальна в $L^{p}(\mathbb{R})$.

ДокАЗАтЕльство. 1) Достаточно показать, что функция $F(z)$ представима в виде $(16)$ с $g(t) \in L^{q}(\mathbb{R})$. В силу теоремы В для этого, в свою очередь, достаточно проверить, что для $G=F$ выполнено условие (18).

Сначала покажем, что при достаточно малом $\delta>0$ конечны части интеграла в (18) с $G=F$, взятые по "секторам" $S_{k}$, введенньм в лемме 5 . Для этого воспользуемся оценкой (36). Получим

$$
\begin{aligned}
& \iint_{S_{k}}|F(z)|^{q}(1+|y|)^{(q-3)(1-\beta / 2)} \exp \left(-q K(\beta, a) r^{\beta}|\sin \theta|^{\beta}\right) d x d y \\
& \quad \leqslant C \iint_{S_{k}} \exp \left(-q K(\beta, a) r^{\beta} b(\theta)\right) r^{(q-3)(1-\beta / 2)+q \beta(1-\operatorname{Re} \mu)+1} d r d \theta .
\end{aligned}
$$

По условию $\operatorname{Re} \mu>1 / \beta+1 /(q \alpha)+1 / 2$, и значит, $(q-3)(1-\beta / 2)+q \beta(1-\operatorname{Re} \mu)+$ $2<\beta / 2$. По лемме 5 интеграл (39) конечен, $k=\overline{1, m}$.

Таким образом, остается рассмотреть часть интеграла (18) с $G=F$, взятую по множеству

$$
\left(z: z \in \mathbb{C}, r>1, z \notin \bigcup\left(S_{k}: k=\overline{1, m}\right)\right) .
$$

В ходе доказательства леммы 1 мы показали, что все отрезки $\bar{I}_{k}, k=\overline{1, m}$, лежат на множестве $(-\pi, 0) \cup(0, \pi)$, т.е. при некотором $\gamma>0$

$$
\bigcup\left(\bar{I}_{k}: k=\overline{1, m}\right) \subseteq(\theta: \gamma \leqslant|\theta| \leqslant \pi-\gamma)
$$

Пусть $b=\max (\beta(1-\operatorname{Re} \mu),-1)$. Тогда по теореме $\mathrm{A}$

$$
|F(z)| \leqslant C r^{b} \exp \left(K(\beta, a) r^{\beta} h(\theta)\right), \quad C>0, \quad r>r_{0}, \quad \gamma \leqslant|\theta| \leqslant \pi-\gamma,
$$

и значит,

$$
\begin{aligned}
& |F(z)|^{q}(1+|y|)^{(q-3)(1-\beta / 2)} \exp \left(-q K(\beta, a) r^{\beta}|\sin \theta|^{\beta}\right) \\
& \quad \leqslant C r^{l} \exp \left(-q K(\beta, a) r^{\beta} b(\theta)\right), \quad r>r_{0}, \quad \gamma \leqslant|\theta| \leqslant \pi-\gamma,
\end{aligned}
$$

при некотором $l$. По лемме $2 b(\theta) \geqslant \varepsilon>0$ вне объединения окрестностей $U_{k}$ точек $\theta_{k}, k=\overline{1, m}$. Значит, если

$$
P=\left(\theta: \gamma \leqslant|\theta| \leqslant \pi-\gamma, \theta \notin \bigcup\left(U_{k}: k=\overline{1, m}\right)\right),
$$


то при некотором $\varepsilon_{1}>0$

$$
|F(z)|^{q}(1+|y|)^{(q-3)(1-\beta / 2)} \exp \left(-q K(\beta, a) r^{\beta}|\sin \theta|^{\beta}\right) \leqslant C \exp \left(-\varepsilon_{1} r^{\beta}\right)
$$

для $\theta \in P, r>r_{1}$. Это показывает, что часть интеграла в $(18)$ с $G=F$, распространенная на множество

$$
\left(z: r>1, \gamma \leqslant|\theta| \leqslant \pi-\gamma, \theta \notin \bigcup U_{k}\right)
$$

конечна. Остается рассмотреть часть интеграла (18) с $G=F$, взятую по множесTBy

$$
\sigma=(z: r>1,(|\theta|<\gamma) \cup(\pi-\gamma \leqslant|\theta| \leqslant \pi)
$$

По теореме $\mathrm{A}|F(z)|=O\left(r^{-1}\right), z \in \sigma$, и значит, на $\sigma$

$$
|F(z)|^{q}(1+|y|)^{(q-3)(1-\beta / 2)} \exp \left(-q K(\beta, a)|y|^{\beta}\right) \leqslant C(1+|y|)^{s} r^{-q} \exp \left(-A|y|^{\beta}\right) .
$$

Отсюда и из леммы 6 следует конечность той части интеграла в $(18)$ с $G=F$, которая взята по множеству $\sigma$. В итоге мы показали, что для функции $G=F$ условие (18) выполнено. Утверждение 1) доказано.

2) Сначала покажем, что

$$
\iint_{r>1}|F(z)|^{q} r^{-q}(1+|y|)^{(q-3)(1-\beta / 2)} \exp \left(-q K(\beta, a)|y|^{\beta}\right) d x d y<\infty .
$$

В ходе доказательства утверждения 1) мы проверяли условие (18) для $G=F$. Теперь в (40) появился дополнительный множитель $r^{-q}$. Поэтому нам достаточно проследить, какие изменения в доказательство вносит этот множитель.

Очевидно, что множитель $r^{-q}$ не повлияет на части интеграла, берущиеся по множествам $\sigma$ и $(z: r>1, \theta \in P)$. Надо только учесть влияние этого множителя в интегралах по “секторам" $S_{k}$.

Используя оценку (36), имеем

$$
\begin{aligned}
& \iint_{S_{k}}|F(z)|^{q} r^{-q}(1+|y|)^{(q-3)(1-\beta / 2)} \exp \left(-q K(\beta, a)|y|^{\beta}\right) d x d y \\
& \quad \leqslant C \iint_{S_{k}} \exp \left(-q K(\beta, a) r^{\beta} b(\theta)\right) r^{(q-3)(1-\beta / 2)+q \beta(1-\operatorname{Re} \mu)+1-q} d r d \theta .
\end{aligned}
$$

По условию $\operatorname{Re} \mu>1 /(q \alpha)+1 / 2$. Значит, $(q-3)(1-\beta / 2)+q \beta(1-\operatorname{Re} \mu)+2-q<\beta / 2$, и по лемме 5 интеграл (41) конечен. Мы доказали свойство (40).

Перейдем к непосредственному доказательству минимальности системы (1). Предположим сначала, что все нули $\lambda_{n}(n \in \mathbb{N})$ функции $F(z)$ просты. Из (40) следует, что для целой функции

$$
\frac{F(z)}{z-\lambda_{n}}
$$

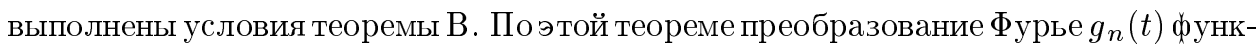
ции $(42)$ лежит в $L^{q}(\mathbb{R})$. Но функции

$$
h_{n}(t)=\frac{g_{n}(t)}{F^{\prime}\left(\lambda_{n}\right)}, \quad n \in \mathbb{N}
$$


образуют биортогональную систему к системе $(1)$ на $\mathbb{R}$. По доказанному $h_{n}(t) \in$ $L^{q}(\mathbb{R})$, и значит, система $(1)$ минимальна в $L^{p}(\mathbb{R})$.

Пусть теперь $\lambda_{n}-$ корень кратности $m_{n}$ функции $F(z)$. Тогда точке $\lambda_{n}$ в системе (1) отвечают $m_{n}$ функций

$$
e^{-i \lambda_{n} t}, t e^{-i \lambda_{n} t}, \ldots, t^{m_{n}-1} e^{-i \lambda_{n} t} .
$$

В этом случае соответствующий набор

$$
h_{n, 1}(t), h_{n, 2}(t), \ldots, h_{n, m_{n}}(t)
$$

функций биортогональной системы состоит из подходящих линейных комбинаций преобразований Фурье функций

$$
\frac{F(z)}{z-\lambda_{n}}, \frac{F(z)}{\left(z-\lambda_{n}\right)^{2}}, \ldots, \frac{F(z)}{\left(z-\lambda_{n}\right)^{m_{n}}}
$$

(см. [13]). Мы уже доказали, что для первой функции (44) выполнены условия теоремы В. Подавно они выполнены и для остальных функций (44). Значит, функции (43) лежат в $L^{q}(\mathbb{R})$. Снова все функции биортогональной системы к системе (1) на $\mathbb{R}$ принадлежат пространству $L^{q}(\mathbb{R})$, т.е. система $(1)$ минимальна в $L^{p}(\mathbb{R})$. Теорема 3 доказана.

ТЕОРема 4. Пусть функиия $F(z)$ та же, что в теореме 2, пусть $\Lambda$ последовательность всех нулей функиии $F(z)$. Пусть $2 \leqslant p<\infty$. Тогда

1) если выполнено условие (37), то система (1) неполна в $L^{p}\left(\mathbb{R}_{+}\right)$,

2) если выполнено условие (38), то система (1) минимальна в $L^{p}\left(\mathbb{R}_{+}\right)$.

ДокАЗАтЕльство. 1) Достаточно показать, что функция $F(z)$ представима в виде $(16)$ с $g(t) \in L^{q}(\mathbb{R}), g(t)=0, t<0$. По теореме В для этого достаточно проверить, что $F(z) \in H_{-}^{2}$ и что для $G=F$ конечен интеграл в $(18)$, взятый по верхней полуплоскости $y>0$. Конечность этого интеграла устанавливается так же, как в доказательстве теоремы 3 . Далее, теперь $\theta_{k} \in(0, \pi)$, и значит (см. доказательство леммы 1$)$, все интервалы $I_{k}$ также принадлежат $(0, \pi)$. Таким образом, оценки (31), (32) верны для нижней полуплоскости $y<0$. Из них с учетом формулы (22) и следует принадлежность $F(z) \in H_{-}^{2}$. У тверждение 1) доказано. Внося аналогичные изменения в доказательство утверждения 2) теоремы 3 , получаем утверждение 2) теоремы 4 . Теорема 4 доказана.

СлЕДСТВИЕ. Пусть $\Lambda$ - последовательность точек из теоремы 1 (теоремы 2). Пусть $2 \leqslant p<\infty$. Тогда система (1):

1) полна в $L^{p}(\mathbb{R}) \quad\left(L^{p}\left(\mathbb{R}_{+}\right)\right)$тогда и только тогда, когда выполнено условие (23),

2) минимальна в $L^{p}(\mathbb{R})\left(L^{p}\left(\mathbb{R}_{+}\right)\right)$тогда и только тогда, когда выполнено условие (38),

3) полна и минимальна в $L^{p}(\mathbb{R})\left(L^{p}\left(\mathbb{R}_{+}\right)\right)$тогда и только тогда, когда

$$
\frac{1}{q \alpha}+\frac{1}{2}<\operatorname{Re} \mu \leqslant \frac{1}{\beta}+\frac{1}{q \alpha}+\frac{1}{2} .
$$


5. Присутствуюшие в теоремах 1-4 функции $F(z)$ являются подходящими линейными комбинациями функций типа Миттаг-Леффлера. Если $1<\beta \leqslant 2$, то в теореме 2 можно взять всего одну точку $\theta_{k}$, т.е. в этом случае $F(z)$ совпадает с функцией типа Миттаг-Леффлера. Асимптотика нулей таких функций изучена достаточно полно (см. [11], [14]). Но в условиях теоремы 1 число слагаемых соответствующей линейной комбинации не меньше двух. В связи с доказанными теоремами вопрос о распределении нулей функций $F(z)$ нуждается в исследовании, но для этого потребуется отдельная работа.

\section{Список литературы}

1. Zalik $R$. On approximation by shifts and a theorem of Wiener // Trans. Amer. Math. Soc. 1978. V. 243. P. 299-308.

2. Zalik $R$. On some gap theorems and the closure of translates // Notices Amer. Math. Soc. 1978. V. 25. № 2. P. A-314.

3. Faxen $B$. On approximation by translates and related problems in function theory // Ark. Math. 1981. V. 19. № 2. P. 271-289.

4. Седлецкий A. M. Аппроксимация сдвигами и полнота взвешенных систем экспонент в $L^{2}(\mathbb{R}) / /$ Матем. сб. 1984. Т. 123. № 1. С. 92-107.

5. Седлецкий A.M. Аппроксимация сдвигами функции на прямой // Теория приближ. функций. Тр. Междунар. конф. приближ. функций, Киев, 31 мая - 5 июня, 1983. М.: Наука, 1987. С. 397-400.

6. Винер H. Интеграл Фурье и некоторые его приложения. М.: Физматгиз, 1963.

7. Седлецкий A.M. Аппроксимативные свойства систем экспонент в $L^{p}(a, b) / /$ Дифференц. уравн. 1995. Т. 31. № 10. С. 1675-1681.

8. Zalik R., Abuabara Saad T. Some theorems concerning holomorphic Fourier transforms // J. Math. Anal. Appl. 1987. V. 126. P. 483-493.

9. Сальникова T. А. Полные и минимальные системы экспонент в пространствах $L^{p}(\mathbb{R}) / /$ Матем. заметки. 1994. Т. 55. №3. С. 118-129.

10. Левин Б. Я. Целые функции (курс лекций). М.: Изд-во МГУ, 1971.

11. Джсрбашян M. М. Интегральные преобразования и представления функций в комплексной области. М.: Наука, 1966.

12. Седлецкий A. М. Преобразования Фурье быстро убьвающих функций // Изв. РАН. Сер. матем. 1997. Т. 61. №3. C. 187-202.

13. Седлецкий A. М. Биортогональные разложения функций в ряды экспонент на интервалах вещественной оси // УМН. 1982. Т. 37. № 5. С. 51-95.

14. Седлецкий A. M. Асимптотические формулы для нулей функции типа Миттаг-Лефффлера // Anal. Math. 1994. T. 20. C. 117-132.

Московский государственньй

Поступила в редакцию

университет им. М.В. Ломоносова

30.05.1997 